\title{
Identification of a new locus conferring antixenosis to the brown planthopper in rice cultivar Swarnalata (Oryza sativa L.)
}

\author{
Y.F. Qiu, L. Cheng, F. Liu and R.B. Li \\ Corresponding author: R.B. Li \\ E-mail: lirongbai@126.com \\ Genet. Mol. Res. 12 (3): 3201-3211 (2013) \\ Received October 23, 2012 \\ Accepted February 19, 2013 \\ Published August 29, 2013 \\ DOI http://dx.doi.org/10.4238/2013.August.29.4
}

State Key Laboratory for Conservation and Utilization of Subtropical Agro-Bioresources, Agricultural College, Guangxi University, Nanning, China

\begin{abstract}
The brown planthopper [Nilaparvata lugens (Stål); BPH] has caused severe damage to rice production. The identification of resistance genes and the development of BPH-resistant varieties are economical and effective ways to manage this pest. Using an $\mathrm{F}_{2}$ population from a cross between the Indica cultivars 93-11 and Swarnalata, we mapped the Qbph8 locus to a $7.3-\mathrm{cM}$ region on chromosome 8 in two tests, flanked by the markers RM339 and RM515. In this population, $Q b p h-8$ explained 7.7 and $6.6 \%$ of the phenotypic variance of $\mathrm{BPH}$ preference in both tests. In the BPH host choice test, the average number of settled BPHs on the Qbph-8 plants was less than that on the 93-11 plants over the 24- to 120$\mathrm{h}$ observation period. Furthermore, less BPH insects were observed on the $B P H 6+Q b p h-8$ plant compared with the $B P H 6$ plant or $Q b p h-8$ plant, indicating a stronger antixenotic effect shown in the gene pyramiding plants. Hence, this locus can be pyramided with other BPH resistance genes and applied to breed-resistant varieties, which possibly can improve the resistance level and durable resistance to the $\mathrm{BPH}$.
\end{abstract}

Key words: Brown planthopper [Nilaparvata lugens (Stål)]; Oryza sativa; Gene mapping; bph resistance gene; Antixenosis 


\section{INTRODUCTION}

The brown planthopper [Nilaparvata lugens (Stål); BPH] is one of the most destructive pests to rice production. It can suck sap from the rice plant phloem, as well as transmit several kinds of viruses (Cha et al., 2008). Heavy BPH infestation can cause extensive damage to the rice plants, which would become completely dried and present a "hopper burn" phenomenon in the rice field (Soggawa, 1982). In the past two decades, infestations of planthoppers (main BPH) have intensified across Asia, resulting in heavy yield losses (Normile, 2008). Conventionally, $\mathrm{BPH}$ is managed mainly by application of chemical pesticides, which are expensive in terms of cost and environment in rice production practices. Moreover, pesticides would kill BPH predators, and over-use of pesticides prompts the insect to evolve resistance, which in turn leads to pest resurgence. Therefore, application of pesticides is not an ideal strategy to manage this insect pest. In contrast, the breeding and cultivation of BPH-resistant varieties is the most economical and environmentally friendly strategy to control this insect. Hence, it is imperative to identify BPH resistance genes and incorporate them into rice cultivars to develop resistant varieties. To date, at least 27 major BPH resistance genes have been identified from cultivars or wild rice species (http://www.shigen.nig.ac.jp/rice/oryzabase_submission/gene_nomenclature/). Among the major genes detected, most of them have been located on the rice chromosomes using molecular approaches, and several genes have been applied extensively in the practical rice breeding programs. For instance, the genes Bphl, bph2, Bph3, and $b p h 4$ have been used extensively in Southeast Asia countries, e.g., Thailand (Jairin et al., 2007). For the identified major BPH resistance genes mentioned above, a number of polygenes or quantitative trait loci (QTLs) have been identified in some rice cultivars or wild species. Su et al. (2002) reported three QTLs associated with quantitative resistance to $\mathrm{BPH}$ on chromosomes 2, 10, and 12, using a backcross inbred population derived from Nipponbare x Kasalath. Moreover, by analyzing the mapping population derived from the BPH-resistant landrace Yagyaw and susceptible cultivar Cpslo17, four QTLs (Qbph-2, Qbph-4,Qbph-7, and Qbph-9) accounting for 5.64 to $12.77 \%$ of the phenotypic variation for BPH resistance were identified in the study performed by Liu et al. (2009). In brief, several quantitative resistance QTLs have been detected in rice cultivars or wild species, and some of them have been preliminarily located to a region on the chromosomes. However, as the genetic effects of the detected minor QTLs were too small, few studies were conducted to characterize a single minor QTL, or when it was pyramided with other major resistance genes.

Generally, plants would be attacked by different kinds of exotic insects or pathogens and be associated with certain injuries or diseases. However, the insects themselves may employ different resistance types against the exotic impairment. Traditionally, these resistance types include the following: antixenosis (non-preference), involving a reduction in colonization or oviposition; antibiosis, involving a reduction in insect survival, growth rate, or reproduction after ingestion of host tissue; and tolerance, involving production of a crop of high quality and yield despite insect infestation (Painter, 1951; Alam and Cohen, 1998). Previous studies have shown that these resistance types were conferred by the major BPH resistance gene. Du et al. (2009) detected that Bph14 appeared to only confer antibiosis in transgenic lines. Moreover, Qiu et al. (2012) found that BPH6 and BPH12 conferred both antixenotic and antibiotic effects in near-isogenic line (NIL) plants. In addition, several polygenes or QTLs have been identified to be associated with antixenosis, antibiosis, or tolerance in rice cultivars. Soundararajan et al. (2004) detected six QTLs on chromosomes 1, 2, 6, and 7 for BPH resistance by applying RAPD and RFLP markers 
on the double-haploid population IR64 x Azucena. Of these, the QTL on chromosome 7 was associated with seedling resistance and the QTL on chromosome 2 with antibiosis, whereas QTLs on chromosomes 1, 6, and 7 showed association with tolerance. Ramalingam et al. (2003) reported four additional QTLs associated with BPH resistance in the same population (IR64 x Azucena) using an additional 105 candidate gene markers. These studies also indicated the need to extend the QTL analysis to different growth stages of the plant and over different environments.

It is extensively known that pyramiding major BPH resistance genes can significantly improve resistance levels (Myint et al., 2012; Qiu et al., 2012). Moreover, compared with the single-resistance gene line, rice cultivars could exhibit a more durable BPH resistance when carrying a major BPH resistance gene and several minor resistance QTLs. For example, Cohen et al. (1997) detected that the cultivar IR64 carrying the major gene Bph1 and other minor QTLs directed the cultivar to display a higher resistance level and more durable resistance to the BPH in the field. However, how the resistance is conferred by a single QTL (e.g., QTLassociated antixenosis or tolerance) in a rice cultivar remains unknown.

The rice variety Swarnalata (Indica) has been reported to contain the major resistance gene BPH6 (formerly Bph6) and is resistant to biotype 2 and Bangladesh BPH populations (Kabis and Khush, 1988; Qiu et al., 2010). The BPH6 gene has been mapped to the long arm of chromosome 4, between the single sequence repeat (SSR) markers RM6997 and RM5742, and the BPH6 NILs (BPH6-NILs) expressed an antixenotic effect on the BPH insects (Qiu et al., 2010). In the present study, we aimed to 1) identify whether other resistance loci could confer the antixenosis to the variety Swarnalata at the adult stage in the field, and 2) evaluate the antixenotic level when pyramided with the major BPH resistance gene at the seedling stage in the greenhouse.

\section{MATERIAL AND METHODS}

\section{Plant materials and mapping population}

Swarnalata has been reported to carry the resistance gene $B P H 6$ and is resistant to biotype 2 and Bangladesh BPH populations (Kabis and Khush, 1988; Qiu et al., 2010). Further study showed BPH6 to be located in the region flanked by the markers RM6997 and RM5742 on chromosome 4, and the BPH6-NIL plants showed an antixenotic effect on the BPH insects (Qiu et al., 2010). Rice line 93-11 (Indica) was used as the susceptible parent for the cross. An $\mathrm{F}_{2}$ population consisting of 144 individuals derived from a 93-11 x Swarnalata cross was used to identify and map the antixenosis loci. The $\mathrm{F}_{2}$ individuals were self-pollinated to generate $\mathrm{F}_{3}$ lines, and the homozygous $\mathrm{F}_{2}$ lines at the target region of the BPH6 were selected by using markers RM5742 and RM6997 (Qiu et al., 2010).

\section{BPH settling number survey in the field}

The $F_{2}$ plants, Swarnalata and 93-11, were sown on March 17, 2011 and then transplanted to a field in the Guangxi University in Nanning, China, on April 11, 2011, with eight plants and a distance of $16.5 \mathrm{~cm}$ between plants within a row, and $25 \mathrm{~cm}$ between rows. Two rows of Swarnalata and 93-11 were cultivated on both ends, and three rows were randomly transplanted in the $\mathrm{F}_{2}$ plants. The field management followed essentially the normal agricultural practice. To survey the BPH numbers in the field, we counted the BPH insects settled on 
each plant on June 20 and then on July 19, 2011. The insects, which settled about a $50 \mathrm{~cm}$ high (measuring from the field ground) of all shoots in the $\mathrm{F}_{2}$ population, were surveyed.

\section{Host selection behavior at seedling stage}

For the insect host choice test at seedling stage in the greenhouse, the BPHs were collected from rice fields in 2011 in Nanning, China, and maintained on TN1 (a susceptible Indica variety) plants under natural conditions in a greenhouse at Guangxi University. To perform the test, two 14-day-old seedlings of the $\mathrm{F}_{3}$ and 93-11 were transplanted in a plastic bucket $(17 \mathrm{~cm}$ in diameter, 15 $\mathrm{cm}$ in height) with seedlings of the same genotype at opposite ends of roughly perpendicular diagonals. The bucket was then completely covered with a fine, light-transmitting mesh. The experiment was conducted as described by Qiu et al. (2010) and a total of five buckets were used for each pair of genotypes. To observe the host selection of the BPH insects, 60-s instar nymphs were placed in each bucket and allowed to choose host plants (42 days old), where they would feed and reproduce over a 120-h period. The BPH insects that settled on each plant were counted at 3, 6, 12, 24, 48, 96, and $120 \mathrm{~h}$ after release. Then, the BPH percentage was applied to compare between two genotypes.

\section{DNA extraction, map construction, and QTL analysis}

Total DNA was extracted from fresh leaves of individual plants by the cetyltrimethylammonium bromide method. PCR products were separated on a $6 \%$ denaturing polyacrylamide gel and detected by silver staining. Genomic sequences and SSR markers were obtained from Gramene (http://www.gramene.org/markers/index.html).

Two contrasting DNA bulks were prepared based on the phenotype of the $\mathrm{F}_{2}$ individuals for the bulked segregant analysis (BSA). The bulks consisted of DNA from 10 individuals settling the most or least $\mathrm{BPH}$ number of the $\mathrm{F}_{2}$ population, and were screened with SSRs to identify their link to BPH antixenosis. A local genetic linkage map of SSR markers from the BPH antixenosis gene-containing regions was constructed using JoinMap 3.0 (Van Ooijen and Voorrips, 2001). QTL analysis of the BPH antixenosis was conducted with MapQTL 5.0 (Van Ooijen, 2004).

\section{Statistical analysis}

The chi-square test for goodness-of-fit was performed with MS-Excel. The resistance data were analyzed by using one-way ANOVA and comparing the LSD test at a 5\% significance level by performing with SPSS 13.0 (SPSS Institute Inc., Chicago, IL, USA).

\section{RESULTS}

\section{Genetic analysis of the BPH host settling}

The rice variety Swarnalata has been reported to resist Bangladesh BPH populations (Kabis and Khush, 1988). The major resistance gene BPH6 has been mapped to chromosome 4 between the markers RM6997 and RM5742, and BPH6-NIL plants showed an antixenotic effect on BPHs (Qiu et al., 2010). We also found that significantly more BPH insects fastened themselves onto the susceptible rice line 93-11 seedlings or adult plants, compared with the resistant 
variety Swarnalata, when they were cultivated together in the greenhouse or rice field (data not shown). Therefore, we wanted to detect whether there were other resistance loci conferring BPH antixenosis at the adult stage under natural conditions. In considering this purpose, we only surveyed the settled BPH insects on the $\mathrm{F}_{2}$ individuals at the rice adult stage when the BPHs broke out in the field in this study; we did not investigate $B P H 6$ any further in mapping the resistance loci, because it has been identified and mapped in the previous study using the same mapping population (Qiu et al., 2010). The variety Swarnalata exhibited high antixenosis to the BPH insects, with an average BPH number of 5 in both tests. By contrast, the BPH insects preferred to fasten themselves on the 93-11 plants, and there were more than 100 BPHs settled on each plant when we surveyed in the field. In the $\mathrm{F}_{2}$ population, the settled $\mathrm{BPH}$ number on the individuals showed a continuous range from 3 to 120, and did not show any discrete segregation (Figure 1). The detected average BPH numbers in the $\mathrm{F}_{2}$ population were 18.9 or 20.7 in the first and second tests, respectively (Figure 1). The result indicates that possibly one or more genes/QTLs control the segregation of the settled BPH numbers at the adult stage in the field.

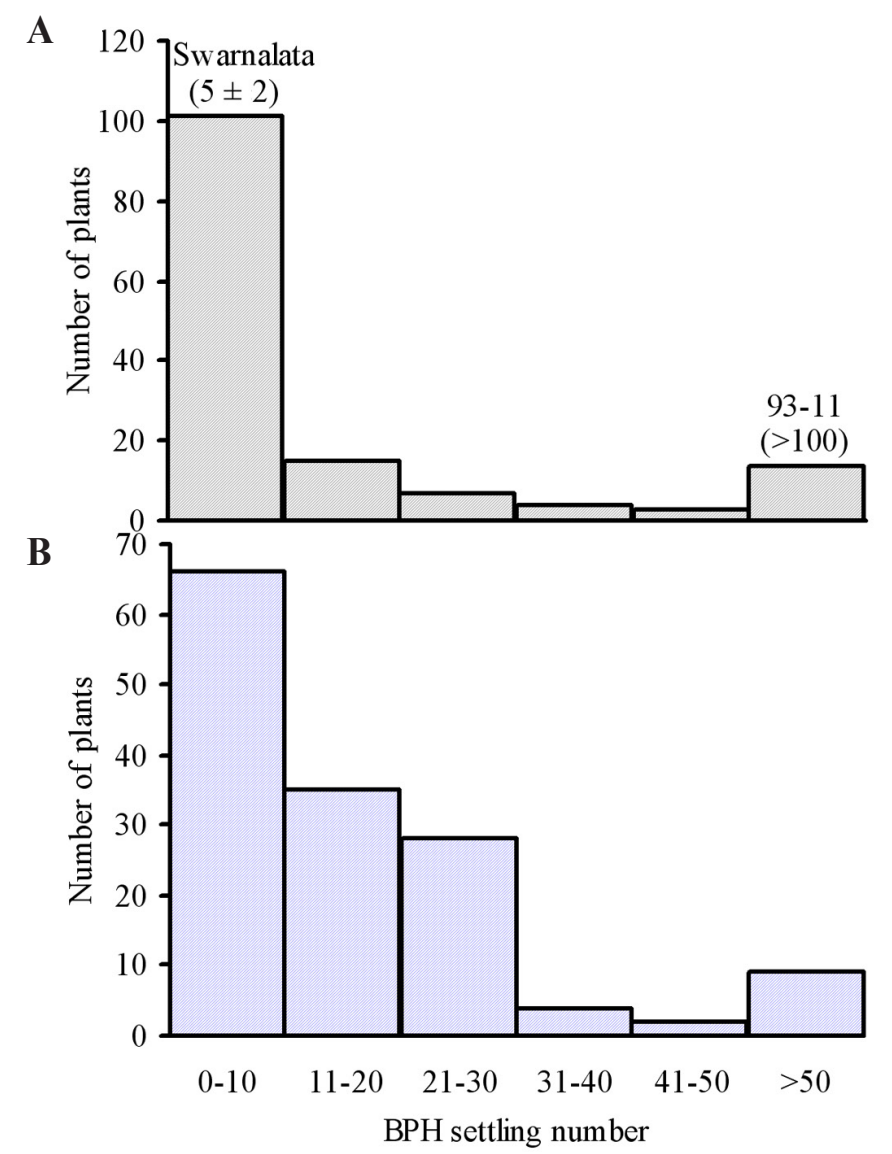

Figure 1. Frequency distribution for the brown planthopper $(\mathrm{BPH})$ settling numbers of an $\mathrm{F}_{2}$ population derived from the cross of 93-11/Swarnalata at adult stage in the field. A. The first counting test. B. The second counting test. The average BPH numbers of the parents Swarnalata and 93-11 plants were 5 and $>100$, respectively. Lower numbers indicate higher antixenosis. 


\section{BPH antixenosis gene mapping}

To identify markers tightly linked to the BPH settling behavior, 520 SSR markers, distributed on 12 rice chromosomes, were used to survey Swarnalata and 93-11. A total of $196(37.7 \%)$ of the markers were polymorphic and were used for BSA. For the RM5742 and RM6997 markers, another two markers (RM339 and RM515), which are all on chromosome 8, were found to differentiate between the two DNA bulks. The region harbored by the RM5742 and RM6997 markers was ignored in the present research as the BPH6 gene conferring antibiosis and antixenosis to the BPHs has been identified in this region (Qiu et al., 2010). Therefore, additional polymorphic markers from chromosome 8 were used to genotype the $144 \mathrm{~F}_{2}$ plants and construct a local linkage map using JoinMap 3.0 (Table 1). The map covered $41 \mathrm{cM}$ of chromosome 8 , and the marker order was basically in agreement with the previously published maps (Temnykh et al., 2000; McCouch et al., 2002). To detect the location of the antixenosis gene, we analyzed the settled BPH numbers and genotypes of the $\mathrm{F}_{2}$ plants by interval mapping using MapQTL 5.0. Consequently, one locus with the largest likelihood (LOD) score for BPH preference was detected between RM339 and RM515 on the short arm of the chromosome 8 in both tests. The locus identified in the first test had the largest LOD score of 2.35, and the genetic distances were $4.1 \mathrm{cM}$ to RM339 and $3.2 \mathrm{cM}$ to RM515. Similarly, it had the largest LOD score of 2.14, and the genetic distances were $4.8 \mathrm{cM}$ to RM339 and $2.5 \mathrm{cM}$ to RM515 in the second test. Variation at this locus explained 7.7 and $6.6 \%$ of the phenotypic variance of $\mathrm{BPH}$ preference in the $\mathrm{F}_{2}$ population in both tests (Table 1). Based on the QTL analysis, the additive effects at the target locus in both tests were -4.8 and 6.9 , respectively, whereas the dominant effects were -21.0 and -9.2 , respectively (Table 1). As the detected locus was a minor QTL and conferred the antixenosis (settling) to the BPH, it was preliminarily designated as $Q b p h-8$.

\begin{tabular}{|c|c|c|c|c|c|c|c|c|}
\hline \multirow{2}{*}{$\begin{array}{l}\text { Locus } \\
\text { RM310 }\end{array}$} & \multirow{2}{*}{$\begin{array}{c}\text { Position }(\mathrm{cM}) \\
0\end{array}$} & \multirow{2}{*}{$\frac{\mathrm{LOD}}{0.47^{\mathrm{b}}}$} & \multicolumn{2}{|c|}{ PEV (\%) } & \multicolumn{2}{|c|}{$A$} & \multicolumn{2}{|c|}{$D$} \\
\hline & & & 0.11 & 1.4 & -2.5 & -1.9 & -7.1 & -0.2 \\
\hline RM331 & 18.6 & 0.89 & 0.19 & 3.6 & -3.0 & -1.8 & -12.4 & -2.7 \\
\hline RM339 & 25.4 & 1.48 & 0.54 & 6.4 & -3.5 & -1.6 & -17.7 & -6.5 \\
\hline Qbph-8 & $29.5(30.2)^{\mathrm{a}}$ & 2.35 & 2.14 & 7.7 & -4.8 & 6.9 & -21.0 & -9.2 \\
\hline RM515 & 32.7 & 2.06 & 1.96 & 7.3 & -4.7 & 6.9 & -21.6 & -8.5 \\
\hline RM284 & 40.8 & 1.29 & 1.26 & 3.9 & 10.2 & 6.5 & 5.2 & -1.5 \\
\hline
\end{tabular}

The loci position and genetic distance were estimated on the progeny genotypes by JoinMap 3.0 and the genetic effect was estimated on the progeny data by MapQTL 5.0. The additive effect was equal to half of the trait value difference between two homozygotes and the dominant effect was equal to the trait value difference between the heterozygote and the middle value of two homozygotes. $\mathrm{PEV}=$ percentage of total phenotypic variance explained by the locus; $A$ = additive effect of the Swarnalata allele; $D=$ dominant effect of the Swarnalata allele. ${ }^{\text {NNumbers }}$ indicate the position with the largest LOD score detected in the two tests. ${ }^{\text {NNumbers }}$ in this row indicate the LOD scores of the first BPH settling number survey and the other row indicates the BPH numbers detected in the second test. The rows of $P E V, A$, and $D$ were similar to the LOD.

\section{Antixenotic effect of $Q b p h-8$ toward the BPH insects}

To test the antixenosis in the BPH host preference conferred by $Q b p h-8$, the homozygous $\mathrm{F}_{3}$ lines at the target region of $Q b p h-8$ (not carrying BPHO) were selected with the 
located markers RM339 and RM515, and used to observe the host choice by the insects in the greenhouse. In the BPH host choice test, the average number of settled BPHs on the Qbph-8 plants was smaller than that of the 93-11 plants, except for the 6-h observation time point, and it remained relatively constant over the 120 -h observation period on both genotype plants (Figure 2). However, one-way ANOVA showed that there was no significant difference in BPH preference for the $Q b p h-8$ plant and 93-11 after the 120-h observation period ( $\mathrm{F}=3.21$, $\mathrm{P}=0.11$ at $3 \mathrm{~h} ; \mathrm{F}=0.04, \mathrm{P}=0.85$ at $6 \mathrm{~h} ; \mathrm{F}=0.05, \mathrm{P}=0.82$ at $12 \mathrm{~h} ; \mathrm{F}=0.65, \mathrm{P}=0.44$ at $24 \mathrm{~h}$; $\mathrm{F}=2.75, \mathrm{P}=0.14$ at $48 \mathrm{~h} ; \mathrm{F}=0.12, \mathrm{P}=0.73$ at $96 \mathrm{~h} ; \mathrm{F}=1.01, \mathrm{P}=0.34$ at $120 \mathrm{~h}$ ).

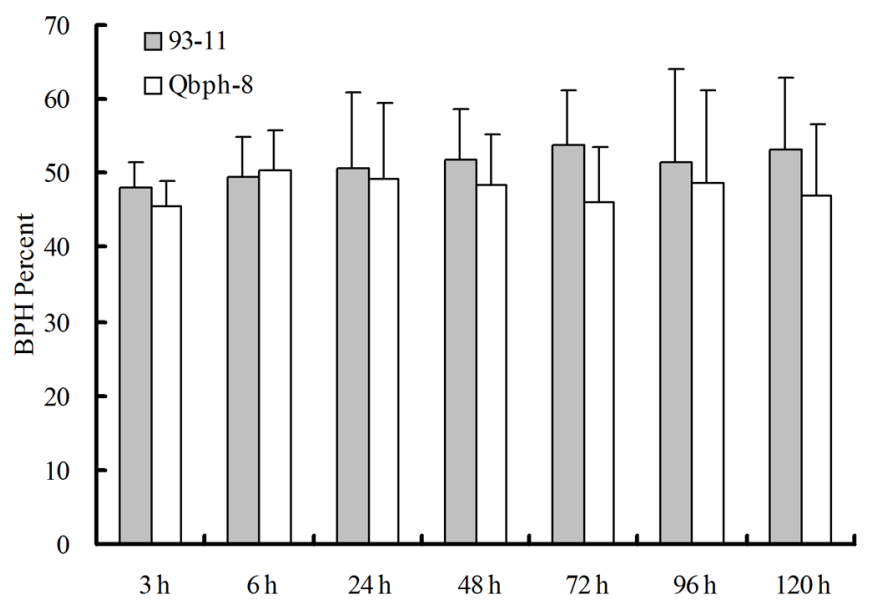

Figure 2. Results of brown planthopper (BPH) host choice test between $Q b p h-8$ plants and 93-11 at the seedling stage in the greenhouse. Bars represent means of five replicates. Error bars represent the SD.

To detect the antixenotic effects conferred by the single locus $Q b p h-8, B P H 6$, or the pyramiding gene, the homozygous $\mathrm{F}_{3}$ lines were used to observe the host choice by the insects in the greenhouse. As shown in Figure 3A, the average numbers of settled BPHs on the BPH6 plant were less than those on the 93-11 plant. One-way ANOVA showed that the BPH insects had significant preference for the 93-11 plant from the observation period of 24 to 120 $\mathrm{h}(\mathrm{F}=31.1, \mathrm{P}=0.005$ at $24 \mathrm{~h} ; \mathrm{F}=34.3, \mathrm{P}=0.004$ at $48 \mathrm{~h} ; \mathrm{F}=59.3, \mathrm{P}=0.002$ at $72 \mathrm{~h} ; \mathrm{P}<$ 0.001 at 96 and 120 h). Similarly, the plants carrying both BPH6 and Qbph-8 also exhibited significantly antixenotic effects on the BPH from the observation period of 24 to $120 \mathrm{~h}(\mathrm{P}<$ 0.001 at 24, 48, 72, 96, and $120 \mathrm{~h}$; Figure 3B). Generally, less BPHs were observed on the $B P H 6+Q b p h-8$ plant compared with the $B P H 6$ plant, indicating a stronger antixenotic effect in the gene pyramiding plants. Further host choice test results between these two genotype plants were also in accordance with this consequence. When we tested the host choice between the $B P H 6+Q b p h-8$ plants and BPH6 plants, less BPH insects were observed on the gene pyramiding plants during the observation period of 6 to $120 \mathrm{~h}$ (Figure 3C). However, no significant difference was shown between them $(\mathrm{F}=0.11, \mathrm{P}=0.76$ at $3 \mathrm{~h} ; \mathrm{F}=0.35, \mathrm{P}=0.58$ at $6 \mathrm{~h} ; \mathrm{F}=$ $0.11, \mathrm{P}=0.76$ at $24 \mathrm{~h} ; \mathrm{F}=0.11, \mathrm{P}=0.75$ at $48 \mathrm{~h} ; \mathrm{F}=0.65, \mathrm{P}=0.45$ at $72 \mathrm{~h} ; \mathrm{F}=1.24, \mathrm{P}=0.31$ at $96 \mathrm{~h} ; \mathrm{F}=2.50, \mathrm{P}=0.17$ at $120 \mathrm{~h}$ ). By contrast, more BPH insects were attached to the $Q b p h-8$ plants in comparison with those on the $B P H 6+Q b p h-8$ plants, and there was a significant dif- 
ference between them when the plants were infested during 24 to $120 \mathrm{~h}(\mathrm{~F}=9.59, \mathrm{P}=0.01$ at $24 \mathrm{~h} ; \mathrm{F}=12.6, \mathrm{P}=0.007$ at $48 \mathrm{~h} ; \mathrm{F}=5.98, \mathrm{P}=0.03$ at $72 \mathrm{~h} ; \mathrm{P}<0.001$ at 96 and $120 \mathrm{~h} ;$ Figure 3D). All these results indicate that $Q b p h-8$ conferred a moderately antixenotic effect on the $\mathrm{BPH}$ insects at the seedling or adult stages, and the antixenosis in the variety Swarnalata was conferred by both BPH6 and Qbph-8.

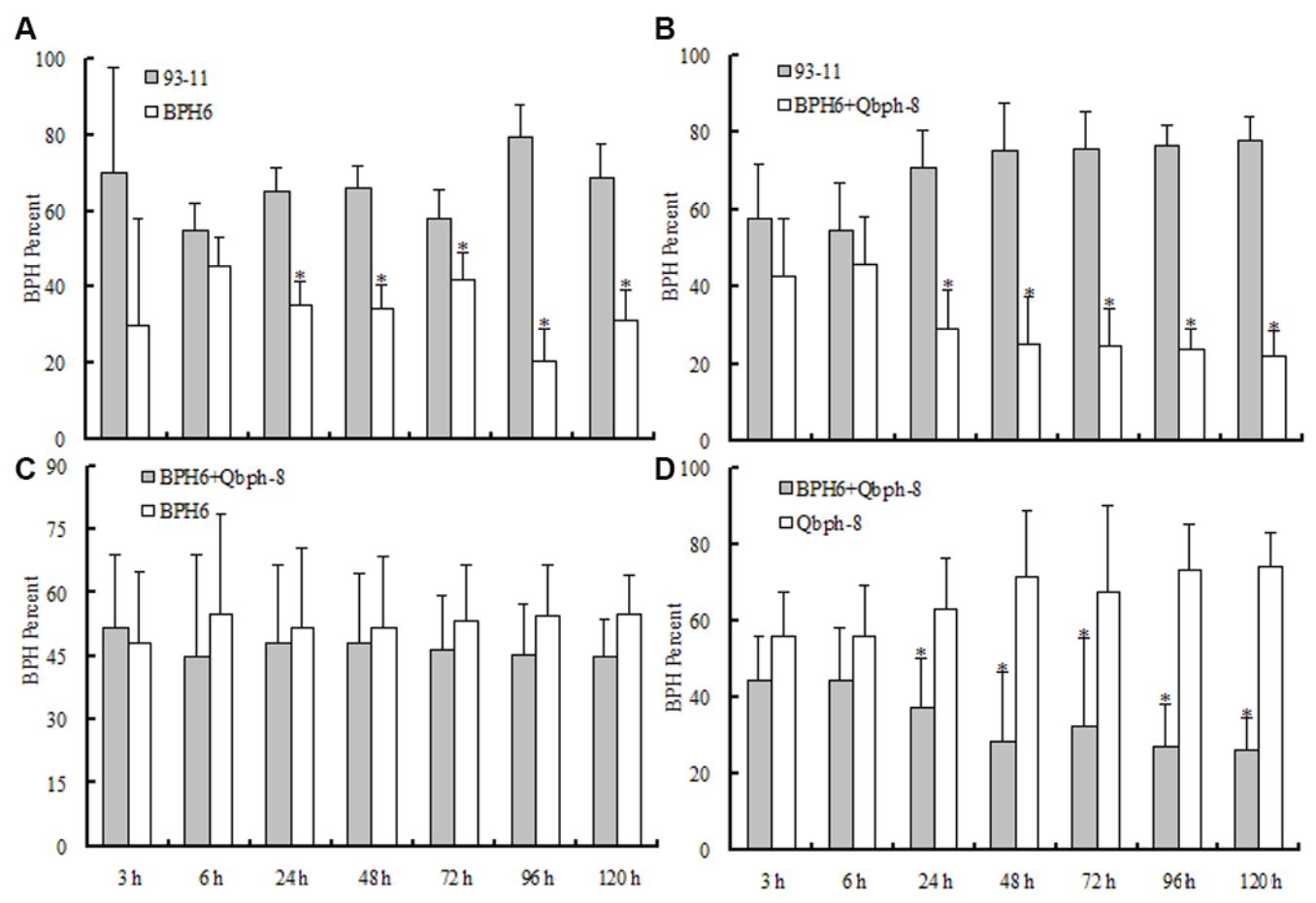

Figure 3. Results of brown planthopper (BPH) host choice test at the seedling stage in the greenhouse. A. 93-11 and $B P H 6$ plants; B. $93-11$ and $B P H 6+Q b p h-8$ plants; C. $B P H 6+Q b p h-8$ plants and $B P H 6$ plants; D. $B P H 6+Q b p h-8$ plants and $Q b p h-8$ plants. Bars represent means of five replicates. Error bars represent the SD. Means labeled with asterisks are considered to be significantly different $(\mathrm{P}<0.05)$.

\section{DISCUSSION}

In the present study, by detecting the BPH numbers settled on the $\mathrm{F}_{2}$ individuals derived from 93-11 x Swarnalata at the adult stage in the field, we have identified one locus that conferred $\mathrm{BPH}$ antixenosis and expressed 7.7 and $6.6 \%$ phenotypic variations in two tests. This locus has been located to the region flanked by markers RM339 and RM515 on chromosome 8. Previously, Alam and Cohen (1998) identified seven QTLs for resistance on chromosomes $1,2,3,4,6$, and 8 using 175 RFLP markers. Of these, one QTL conferring for antixenosis (settling or oviposition) was located to a region between markers amp2 (isozyme) and CDO99 (RFLP) on chromosome 8, using two different BPH populations. This QTL had an LOD score of 2.20 or 2.15 , and expressed a phenotypic variation of 8.1 and $7.4 \%$, respectively, when QTL mapping was performed in the double-haploid lines derived from IR64 x Azucena. Based on 
the reported markers and the Nipponbare or 93-11 genome sequences, $Q b p h-8$ is different to the QTL detected by Alam and Cohen (1998) and should be located to its north. It has been noted that many BPH resistance genes appear to be clustered on rice chromosomes (Jena and Kim, 2010; Qiu et al., 2010; Yara et al., 2010). For example, seven BPH resistance genes [Bph1, bph2, Bph9, Bph10, Bph18(t), bph19(t), and Bph21(t)] cluster in one region between markers G2140 and RM5479 on chromosome 12 based on the sequence of Nipponbare. Another seven genes cluster at two regions on chromosome 4: one that contains BPH12, Bph15, Bph17, and $B p h 20(t)$, and covers about $4.9 \mathrm{Mb}$ between markers C946 and RM5953; and another that includes bph12, bph18(t), and BPH6, spanning $4.1 \mathrm{Mb}$ between markers G271 and RM273. However, no major BPH resistance genes were identified on chromosome 8, and only one QTL described by Alam and Cohen (1998) was detected on this chromosome.

Until now, the genetic basis of qualitative resistance to BPHs has been well established, and at least 27 major genes for resistance to BPHs have been identified from various rice germplasms, including five wild Oryza species (O. officinalis, O. minuta, O. latifolia, O. rufipogon, and $O$. australiensis). In some cases, varieties with single major genes for resistance were easier to be broken down because of the adaptation of $\mathrm{BPH}$ populations to highly resistant varieties. To resolve this problem, progress has been made in pyramiding some of the major BPH resistance genes into elite cultivars with available molecular markers. Li et al. (2006) incorporated Bph14 and Bph15 genes through marker-assisted selection (MAS) into a number of parental lines of hybrid rice in China and observed that 92.3\% Bph14 single-introgression lines had moderate resistance to $\mathrm{BPH}$, and Bph14/Bph15 pyramided lines had stronger resistance levels than the single-gene introgression lines. Qiu et al. (2012) used MAS for pyramiding the BPH6 and $\mathrm{BPH} 12$ genes into a Japonica rice variety, Nipponbare. The pyramided lines showed higher resistance than the lines with a single gene. Moreover, Myint et al. (2012) reported that the pyramided lines carrying $\mathrm{BPH} 25$ and $\mathrm{BPH} 26$ showed high resistance levels. It has been known that pyramiding major resistance genes can significantly improve the varieties' resistance level to the BPH insects, but whether it has more durable resistance remains unknown. By contrast, the rice cultivars with major resistance gene and other minor resistant QTLs present a more durable resistance to the BPH insects (Bosque-Perez and Buddenhagen, 1992). For instance, using 175 RFLP markers, Cohen et al. (1997) detected that the cultivar IR64 carried the major gene $B p h 1$ and seven minor QTLs for the BPH resistance, which directed the cultivar to display a moderate and durable resistance to the BPH in the field. In the present research, less BPH insects were observed on the adult $\mathrm{F}_{2}$ individuals carrying $Q b p h-8$ in the field (Figure 1), or on the $B P H 6+Q b p h-8$ seedlings, compared with the BPH6 or $Q b p h-8$ seedlings in the host choice test conducted in the greenhouse (Figure 3B, C, and D). Since Qbph-8-mediated resistance in rice confers a moderate level of antixenosis, it should impose a relatively moderate selection pressure on the BPH and thereby retain durability in the field.

In comparison with the qualitative resistance, the genetic basis of the quantitative resistance to insect pests was not ascertained owing to the complex inheritance of the trait and the limitations of conventional genetic tools. With the advent of new molecular genetic tools and the availability of ideal mapping populations, it has become possible to look for genes involved in the expression of complex traits. Here, we identified and located the $Q b p h-8$ locus to the region between RM339 and RM515 by analyzing the $\mathrm{F}_{2}$ population with SSR markers. Furthermore, it has been shown that the $\mathrm{F}_{3}$ homozygous lines with $B P H 6$ and $Q b p h-8$ can moderately improve the antixenosis to the BPH insects. Hence, this locus can be pyramided with 
other BPH resistance genes with the located markers and applied to breed-resistant varieties, which possibly can improve the durable resistance to the BPHs. Our study also suggested that the minor resistance genes/QTLs could be identified by performing the traditional mapping strategy, and the resistance mechanisms of the single minor genes/QTLs can be characterized.

\section{ACKNOWLEDGMENTS}

Research supported by the National Natural Science Foundation of China (Grant \#31160276), and the Project Sponsored by the Scientific Research Foundation of Guangxi University (Grant \#1104002).

\section{REFERENCES}

Alam SN and Cohen MB (1998). Detection and analysis of QTLs for resistance to the brown planthopper, Nilaparvata lugens, in a doubled-haploid rice population. Theor. Appl. Genet. 97: 1370-1379.

Bosque-Perez NA and Buddenhagen IW (1992). The Development of Host-Plant Resistance to Insect Pests: Outlook for the Tropics. Proc. 8th Int. Symp. Insect-Plant Relationships (Menken SBJ, Visser JH and Harrewijn P, eds.). Kluwer, Dordrecht.

Cha YS, Ji H, Yun DW, Ahn BO, et al. (2008). Fine mapping of the rice Bphl gene, which confers resistance to the brown planthopper (Nilaparvata lugens Stål), and development of STS markers for marker-assisted selection. Mol. Cells 26: $146-151$.

Cohen MB, Alam SN, Medina EB and Bernal CC (1997). Brown planthopper, Nilaparvata lugens, resistance in rice cultivar IR64: mechanism and role in successful $N$. lugens management in Central Luzon, Philippines. Entomol. Exp. Appl. 85: 221-229.

Du B, Zhang W, Liu B, Hu J, et al. (2009). Identification and characterization of Bph14, a gene conferring resistance to brown planthopper in rice. Proc. Natl. Acad. Sci. U. S. A. 106: 22163-22168.

Jairin J, Phengrat K, Teangdeerith S, Vanavichit A, et al. (2007). Mapping of a broad-spectrum brown planthopper resistance gene, Bph3, on rice chromosome 6. Mol. Breed. 19: 35-44.

Jena KK and Kim SM (2010). Current status of brown planthopper (BPH) resistance and genetics. Rice 3: 161-171.

Kabis MA and Khush GS (1988). Genetic analysis of resistance to brown planthopper in rice (Oryza sativa L.). Plant Breed. 100: 54-58.

Li JB, Xia MY, Qi HX, He GC, et al. (2006). Marker-assisted selection for brown planthopper (Nilaparvata lugens Stål) resistance genes Bph14 and Bph15 in rice. Sci. Agric. Sin. 39: 2132-2137.

Liu Y, Su C, Jiang L, He J, et al. (2009). The distribution and identification of brown planthopper resistance genes in rice. Hereditas 146: 67-73.

McCouch SR, Teytelman L, Xu Y, Lobos KB, et al. (2002). Development and mapping of 2240 new SSR markers for rice (Oryza sativa L.). DNA Res. 9: 199-207.

Myint KK, Fujita D, Matsumura M, Sonoda T, et al. (2012). Mapping and pyramiding of two major genes for resistance to the brown planthopper (Nilaparvata lugens [Stal]) in the rice cultivar ADR52. Theor. Appl. Genet. 124: 495-504.

Normile D (2008). Agricultural research. Reinventing rice to feed the world. Science 321: 330-333.

Painter RH (1951). Insect Resistance in Crop Plants. The Macmillan Company, New York.

Qiu Y, Guo J, Jing S, Zhu L, et al. (2010). High-resolution mapping of the brown planthopper resistance gene Bph6 in rice and characterizing its resistance in the 9311 and Nipponbare near isogenic backgrounds. Theor. Appl. Genet. 121: 1601-1611.

Qiu Y, Guo J, Jing S, Zhu L, et al. (2012). Development and characterization of japonica rice lines carrying the brown planthopper-resistance genes BPH12 and BPH6. Theor. Appl. Genet. 124: 485-494.

Ramalingam J, Vera Cruz CM, Kukreja K, Chittoor JM, et al. (2003). Candidate defense genes from rice, barley, and maize and their association with qualitative and quantitative resistance in rice. Mol. Plant Microbe Interact. 16: 14-24.

Sōgawa K (1982). The rice brown planthopper: Feeding physiology and host plant interactions. Annu. Rev. Entomol. 27: 49-73.

Soundararajan RP, Kadirvel P, Gunathilagaraj K and Maheswaran M (2004). Mapping of quantitative trait loci associated with resistance to brown planthopper in rice by means of a doubled haploid population. Crop Sci. 44: 2214-2220. 
Su CC, Cheng XN, Zhai HQ and Wan JM (2002). Detection and analysis of QTL for resistance to the brown planthopper, Nilaparvata lugens (Stal), in rice (Oryza sativa L.), using backcross inbred lines. Yi Chuan Xue Bao 29: 332-338.

Temnykh S, Park WD, Ayres N, Cartinhour S, et al. (2000). Mapping and genome organization of microsatellite sequences in rice (Oryza sativa L.). Theor. Appl. Genet. 100: 697-712.

Van Ooijen JW (2004). MapQTL 5.0, Software for the Mapping of Quantitative Trait Loci in Experimental Populations (Kyazma BV, ed.). The Netherlands Plant Research International, Wageningen.

Van Ooijen JW and Voorrips RE (2001). JoinMap 3.0, Software for the Calculation of Genetic Linkage Maps (Kyazma $\mathrm{BV}$, ed.). The Netherlands Plant Research International, Wageningen.

Yara A, Phi CN, Matsumura M, Yoshimura A, et al. (2010). Development of near-isogenic lines for $B P H 25(t)$ and $B P H 26(t)$, which confer resistance to the brown planthopper, Nilaparvata lugens (Stål.) in Indica rice 'ADR52'. Breed. Sci. 60: 639-647. 